\title{
Effect of chemical diffusivity on the peak shapes in flow injection analysis with sample or reagent injection
}

\author{
Jinxin $\mathrm{Li}^{\mathrm{a}, *}$, Guojun Liu ${ }^{\text {a }}$, Huichang Ma ${ }^{\mathrm{b}}$, Takashi Korenaga ${ }^{\mathrm{c}}$ \\ ${ }^{a}$ Central Laboratory, Institute of Geophysical and Geochemical Exploration, Langfang, Hebei 102849, China \\ ${ }^{b}$ Research Center for Eco-Environmental Sciences, Academia Sinica, P.O. Box 2871, Beijing 100085, China \\ 'Toyama National College of Technology, 13, Hongo-machi, Toyama 939, Japan
}

Received 6 January 1995; revised 17 February 1995; accepted 26 February 1995

\begin{abstract}
This paper deals with the difference in residence time of the two reagent/sample interface peaks produced in a single channel manifold. This difference in residence time is explained on the basis of a dispersion model, and the relevant factors were studied. It was found that in both sample injection (SI) and reagent injection (RI), in addition to the relative reagent and sample concentrations and injection volumes, the diffusivities of the sample and the reagent will affect the peak shape and detection sensitivity. The sensitivity in peak height measurements and peak area measurements in SI and RI are compared and explained.
\end{abstract}

Keywords: Flow injection; Diffusivity; Overlap coefficient

\section{Introduction}

In flow injection analysis (FIA), there are two injection models which have been used for single channel manifolds: the sample injection model (SI) in which a sample is injected into a reagent or carrier stream [1], and the reagent injection model (RI) in which a reagent is injected into a sample stream [2]. Although it is just a matter of reversing the injection phase, the sensitivity of RI is several times higher than that of SI. Neglecting the chemical reaction between the sample and the reagent, Johnson and Pettey [2] calculated the reagent to sample concentra-

\footnotetext{
* Corresponding author.
}

tion ratio in a single channel manifold by using a "tank-in-series" model. They reported that in RI the maximum concentration of the reagent in the sample has been reduced by a factor 5 and the concentration of the sample in the zone of the reagent has increased from zero to nearly $80 \%$ of its value in the pure carrier. Further mixing will cause an additional increase in the sample concentration in the zone of the reagent, so that RI is more sensitive than SI. In FIA, because the reagent concentration is usually several times higher than the sample concentration, the influence of parameters on the peak shape other than the concentration could hardly be observed. For studying the shapes of SI and RI, Fogg et al. [3] used large injection volumes to produce two separated peaks. They pointed out that the shape of each independent peak depends on the composition and 
relative position of the two solutions forming the boundary layer where the peak is formed. The composition of the solutions and their relative positions in the flow stream affect the shapes and the relative height of the front and the rear peaks. Unfortunately, they did not reveal the relationships between the two peaks, the reason for its formation, and the relationship between the peak position and the sensitivity.

In the present work, the difference in residence time of the front peaks or the rear peaks in the SI and the RI was studied. Results show that the residence time of the reagent/sample interface peak depends mainly on the diffusion coefficients of the reagent and the sample. A dispersion model was used to explain the difference in residence time, and the corresponding detection sensitivity.

\section{Experimental}

\subsection{Reagents}

Reagent grade chemicals were used. Deionized water was used throughout.

\section{1,10-Phenathroline (phen) solution}

A $1.000 \times 10^{-3} \mathrm{M}$ phen stock solution was prepared by dissolving $0.1982 \mathrm{~g}$ phen in about $100 \mathrm{ml}$ of hot (ca. $60^{\circ} \mathrm{C}$ ) water. When the solution was cooled to room temperature, it was diluted to 1000 ml with water. Working solutions were made by diluting this stock solution.

\section{Acetate buffer solution ( $p H$ 4.4)}

A $1 \mathbf{M}$ acetate buffer solution was made by mixing $30 \mathrm{ml}$ of $1 \mathrm{M}$ sodium acetate solution with $70 \mathrm{ml}$ of $1 \mathrm{M}$ acetic acid.

\section{Iron stock solution}

A stock solution of $1.000 \times 10^{-3} \mathrm{M}$ of iron(III) was prepared by dissolving $0.4882 \mathrm{~g}$ ammonium iron sulfate $\left(\mathrm{NH}_{4} \mathrm{Fe}\left(\mathrm{SO}_{4}\right)_{2} \cdot 12 \mathrm{H}_{2} \mathrm{O}\right)$ in $1000 \mathrm{ml}$ of water. A solution containing $2.000 \times 10^{-4} \mathrm{M}$ iron(II) was prepared by mixing $20 \mathrm{ml}$ of the iron stock solution, $10 \mathrm{ml}$ of acetate buffer solution and $2 \mathrm{ml} 1.0 \%$ ascorbic acid solution in a $100 \mathrm{ml}$ volumetric flask and diluting with water.

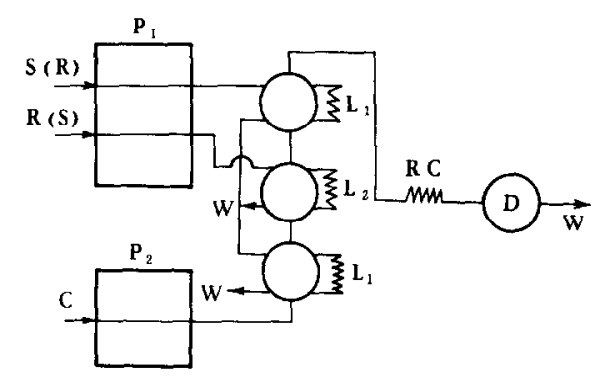

Fig. 1. The manifold used for the study. $P_{1}=$ Peristaltic pump; $P_{2}=$ main pump; $R=6 \times 10^{-4} \mathrm{M}$ phen; $S=2 \times 10^{-4} \mathrm{M}$ iron(II); $\mathrm{C}=$ water carrier; $\mathrm{L}_{1}=$ sample or reagent loop $(200 \mu \mathrm{l}) ; \mathrm{L}_{2}=$ reagent or sample loop $(300 \mu 1) ; \mathrm{RC}=$ reaction coil $(140 \mathrm{~cm} \times 0.5$ $\mathrm{mm}$ i.d.); $\mathrm{D}=$ detector.

\subsection{Apparatus}

A Model K-1000 (Hitachi) flow injection analyzer was used. It contains a 4-channel peristaltic pump, two main pumps and a 16-way valve. A UV-15020 spectrophotometer (Hitachi) equipped with a $8-\mu 1$ flow cell was used as the detector. The sample loop, reagent loop and reaction coil were all made of PTFE tubes (0.5 mm i.d.).

\subsection{Flow system and procedure}

In order to save reagent, a sandwich manifold (Fig. 1) was used. When the SI model was studied, $\mathrm{L}_{1}$ and $\mathrm{L}_{2}$ were loaded with reagent and sample, respectively. In contrast, $L_{1}$ and $L_{2}$ were loaded with sample and reagent, respectively, when the RI model was studied. In both cases, sample and reagent were injected into a water carrier whose flow rate is $0.96 \mathrm{ml} / \mathrm{min}$. The reagent and the sample reacted when they pass through the $140 \mathrm{~cm}$ long reaction coil (RC) and the product was detected at $512 \mathrm{~nm}$.

\section{Results and discussion}

An FIA signal peak consists of two overlapping peaks corresponding to the two reagent/sample interfaces, respectively. If the injection volume is large enough, the two peaks formed at the front and the rear boundaries of the injected bolus can be completely separated, and the effect of the sample or the reagent injection volumes on the shape and the posi- 
tion of the peaks can be neglected. When the concentration ratio of the reagent to the sample is equal to the molar ratio of the complex reaction, the effect of the sample or reagent concentration on the shape and the position of the peaks can be also neglected. For that reason, the effect of the diffusion coefficient is dominant. Fig. 2 shows signal peaks obtained in SI and RI. Fig. 2 also indicates that the distance between the two peaks in SI is larger than in RI. The shape of $P_{1}$ is similar to the shape of $P_{4}$ and both are high and narrow; the shape of $P_{3}$ is similar to that of $P_{2}$ which are lower and broader. The rear peak is always higher than the front peak.

\subsection{Difference in residence time of the front or the rear peaks in the SI and the RI mode}

There is a difference between the residence time $t_{1}$ of the peak $P_{1}$, resulting from the $R \rightarrow S$ (here $R$, $\mathrm{S}$ and $\rightarrow$ denote reagent, sample and chasing direction, respectively) dispersion in SI, and the residence time $t_{3}$ of peak $\mathrm{P}_{3}$, resulting from the $\mathrm{S} \rightarrow \mathrm{R}$ dispersion in RI. The difference can be expressed as $\Delta \Phi_{1}=t_{3}-t_{1}$. Though both $\mathrm{R} \rightarrow \mathrm{S}$ and $\mathrm{S} \rightarrow \mathrm{R}$ entered the system at the same time and passed the same distance with the same flow rate, their peak maxima appeared at different time. Similarly, a difference is observed of the residence time $t_{4}$ of peak $\mathrm{P}_{4}$, resulting from the $\mathrm{R} \rightarrow \mathrm{S}$ diffusion in $\mathrm{RI}$, and the residence time $t_{2}$ of peak $\mathrm{P}_{2}$, resulting from the $\mathrm{S} \rightarrow \mathrm{R}$ diffusion in SI, $\Delta \Phi_{2}=t_{2}-t_{4}$. This means that there is a difference in residence time $(\Delta \Phi)$ in the $\mathrm{R} \rightarrow \mathrm{S}$ and the $\mathrm{S} \rightarrow \mathrm{R}$ dispersions. The $\Delta \Phi_{1}$ and
$\Delta \Phi_{2}$ are generated under different conditions. For $\Delta \Phi_{2}$, the reaction tube is longer $\left(\mathrm{RC}+\mathrm{L}_{1}\right)$, so that the diffusion time is longer, and the difference in residence time resulting from the differences in diffusion are more significant. Therefore, $\Delta \Phi_{2}>\Delta \Phi_{1}$, as shown in Fig. 2, wherein $\Delta \Phi_{1}=0.04 \mathrm{~min}$ and $\Delta \Phi_{2}=0.12 \mathrm{~min}$.

\subsection{Principle of the formation of differences in residence time}

As the chromogenic reagent of this complex reaction is an organic molecule and the concentration of reactants is low, its molecular diffusivity can be calculated using the following equation for nonelectrolyte diffusivity [4].

$E_{A B}=\left(7.4 \times 10^{-8}\left(a M_{\mathrm{B}}\right)^{0.67}\right) /\left(\nu V_{\mathrm{A}}^{0.6}\right)$

Where, $E_{\mathrm{AB}}$ is the diffusivity of the solute $(\mathrm{A})$ in the solvent (B) in $\mathrm{cm}^{2} / \mathrm{s} ; M_{\mathrm{B}}$ is the molecular weight of solvent $\mathrm{B}$ in $\mathrm{g} / \mathrm{mol} ; T$ is the temperature in $\mathrm{K} ; \nu$ is the viscosity of solution in $\mathrm{mPa} ; V_{\mathrm{A}}$ is the molecular volume of solute $A$ at the normal boiling point in $\mathrm{cm}^{3} / \mathrm{mol}$, and $a$ is the association constant.

If the concentration of a solute is low, the viscosity of the solution can be replaced by the viscosity of the solvent (water). From Eq. 1, it can be seen that the diffusivity of the solute in water is inversely proportional to the molecular volume of the solute. The diffusivity of $\mathrm{Fe}^{2+}$ and phen were calculated according to Eq. 1. Results show that $E_{\mathrm{Fe}_{2} \cdot \mathrm{H}_{2} \mathrm{O}}=$ $5.3 E_{\text {phen } \cdot \mathrm{H}_{2} \mathrm{O}}$, which means that the diffusivity of $\mathrm{Fe}^{2+}$ is larger than that of phen.

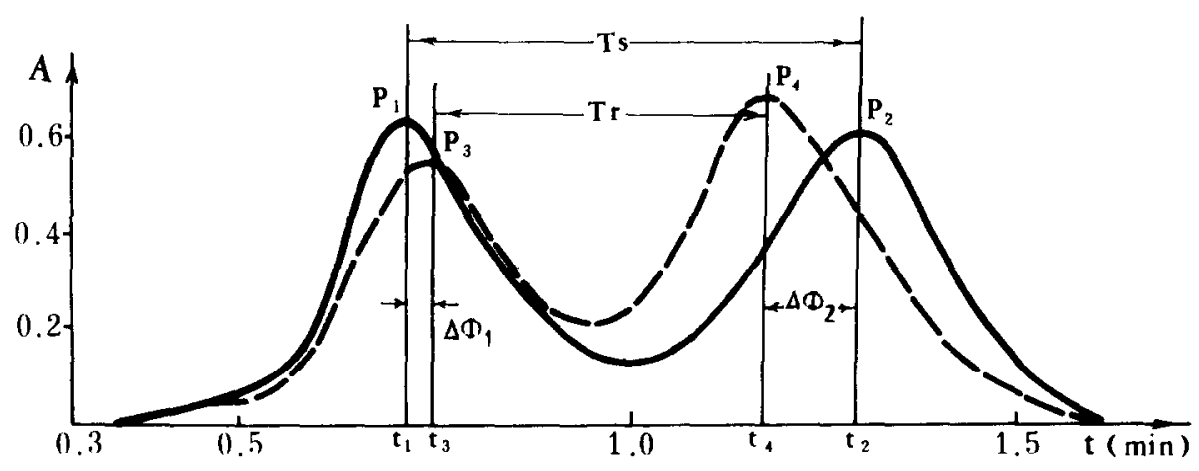

Fig. 2. The peak shapes obtained in SI (solid line) and RI (dashed line). $\mathrm{P}_{i}(i=1-4)=$ peaks formed at the sample/reagent interface; $t_{i}$ $(i=1-4)=$ residence time; $\Delta \Phi_{1}, \Delta \Phi_{2}$ : difference in residence time; $T r, T s$ : distance between the two peaks. 


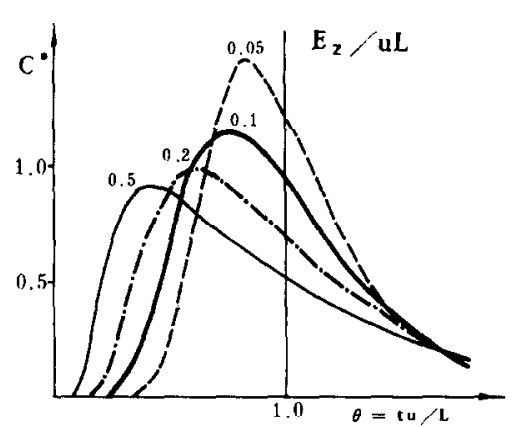

Fig. 3. $E$ curves obtained in a closed vessel under different $E z / u L$ values.

Assuming that the radial dispersion is negligible, the axially dispersed plug flow model was used to describe the single channel flow injection system to permit a greater mathematical simplicity. Using dimensionless concentrations, the general form of partial differential equations representing the dispersion can be shown as follows $[5,6]$.

$$
\begin{aligned}
& \mathrm{d} c^{*} / \mathrm{d} \theta=(E z / u L) \times \mathrm{d}^{2} c^{*} / \mathrm{d} l^{2}-\mathrm{d} c^{*} / \mathrm{d} l \\
& \theta=t u / L
\end{aligned}
$$

in which $c^{*}$ is the dimensionless concentration, $E z$ is the axial dispersion coefficient, $u$ is the flow velocity, $L$ is the tube length. The dimensionless group $(E z / u L)$ is called the vessel dispersion number. The $E$ curve for a closed vessel in an ideally stirred tank is shown in Fig. 3, which indicates that the curve maxima decreases and occurs at lower value with an increase of $E z / u L$. In a pipe where the reagent and sample pass through, the axial mixing is mainly due to fluid velocity gradients, while the radial mixing is due to molecular diffusion only. The $E z$ is influenced by the flow velocity $(u)$, the pipe diameter and molecular diffusivity $\left(E_{\mathrm{AB}}\right)$. The molecular diffusion strongly affects the rate of dispersion in laminar flow, the $E z$ is equal to the $E_{\mathrm{AB}}$ when the dispersion is dominated by diffusion [5]. Because the molecular diffusivity of the reagent is smaller than that of the sample, the reagent dispersion coefficient is small, Ezr $<E z s$. As $L r$ is approximately equal to $L s$, we have $E z r / u L r<$ $E z s / u L s$. At present, each peak of the two reagent/sample interfaces in FIA is studied. In order to visually describe the signal of the product, the product is estimated by the overlap of the reagent

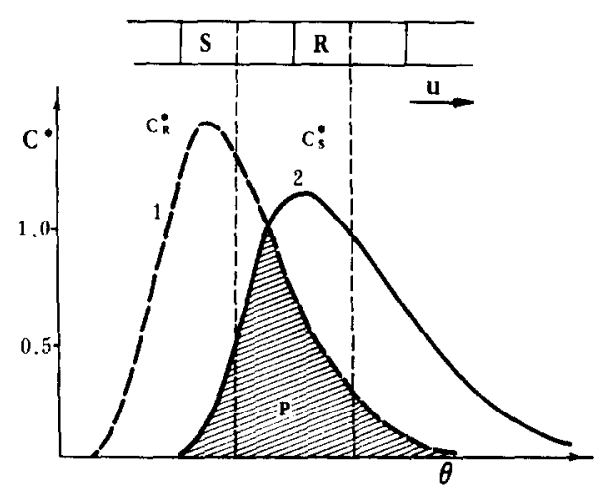

Fig. 4. Distribution curve of the reagent concentration (dashed line) and the sample concentration (solid line) in the $S \rightarrow R$ chasing model. The hatched area represents the reaction product.

and sample concentration distribution. Figs. 4 and 5 show the concentration distribution resulting from the $S \rightarrow R$ and $R \rightarrow S$ chasing model, respectively. The hatched parts in Figs. 4 and 5 denote the distribution of the reaction product. Fig. 4 indicates that in the $S \rightarrow R$ chasing mode the difference of the residence time of $R$ and $S$ was smaller. Therefore, the dispersion of $\mathbf{S}$ and $\mathbf{R}$ was lower and the concentration of the product was higher. On the contrary, the difference of the residence time of $R$ and $S$ in the $R \rightarrow S$ chasing mode was larger (see Fig. 5). Therefore, the dispersion of $R$ and $S$ was larger and the concentration of the product was lower. Comparing Fig. 4 with Fig. 5, it can be concluded that the residence time of the product in the $R \rightarrow S$ chasing model is smaller than that in the $S \rightarrow R$ chasing

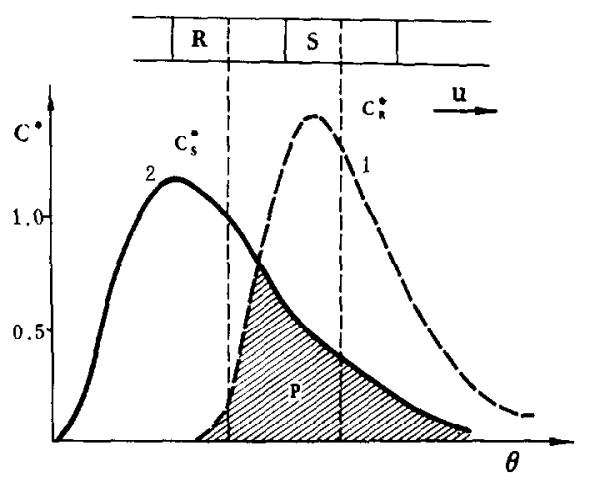

Fig. 5. Distribution curve of the reagent concentration (dashed line) and the sample concentration (solid line) in $R \rightarrow S$ chasing model. The hatched area represents the reaction product. 
model. Therefore, there a difference in residence time exists between the two chasing modes.

\subsection{Relationship between the difference in residence time and the sensitivity}

As mentioned above, there is a difference in residence time in SI and RI. The distance between the front and the rear peaks in SI $\left(T s=t_{2}-t_{1}\right.$, see Fig. 2) and that in $R I\left(T r=t_{4}-t_{3}\right)$ is related with the difference in residence time by the following equation:

$T s=\operatorname{Tr}+\Delta \Phi_{1}+\Delta \Phi_{2}$

where $\Delta \Phi_{1}$ and $\Delta \Phi_{2}$ are the differences in residence time as defined above. With a decrease of the injection volume in FIA, the double peak observed when large injection volumes were used merge and the main peak becomes the signal peak. The peak height of the signal is related to the overlap of the double peak seen in Fig. 6. Since $T r<T s$, the double peaks in RI overlapped more quickly than in SI, and the peak height of the signal in RI is higher than that in SI. According to the above deduction, it can be concluded that the sensitivities of SI and RI are proportional to the difference in residence time. If the peak area is measured in FIA, it represents the total amount of the reaction product, it is not related to the $T$ and $\Delta \Phi$. In this case, the peaks in SI and RI have approximately the same area as long as the measurements are done under the same conditions. The integrated areas, obtained from the absorbance

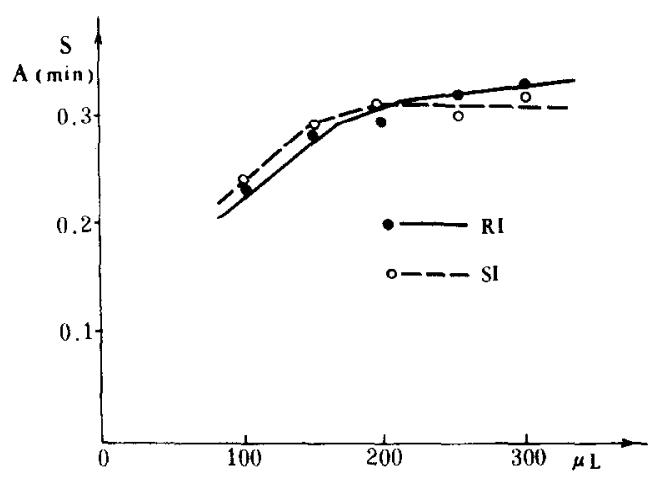

Fig. 7. Effect of injection volumes on the peak area in SI (dashed line) and RI (solid line).

curves in Fig. 6, as a function of injection volume, are shown in Fig. 7. It can be seen from Fig. 7 that both SI and RI have the same sensitivity when peak areas are measured. In both cases, the sensitivity increased with the increase of injection volume. If $S_{1}$ and $S_{2}$ represent the areas of two peaks, $\Delta S$ represents the overlapping area as shown in Fig. 8, an overlap coefficient can be defined as:

$S=\left[2 \Delta S /\left(S_{1}+S_{2}\right)\right] \times 100 \%$

For the peaks which have the same peak height, their difference in residence time can be indicated by $T\left(T=t_{2}-t_{1}\right)$. For the purpose of simple calculation, it is assumed that each peak can be described by the following equation:

$$
A=A_{0} \exp \left[\left(t-t_{\max }\right) / \sigma\right]
$$

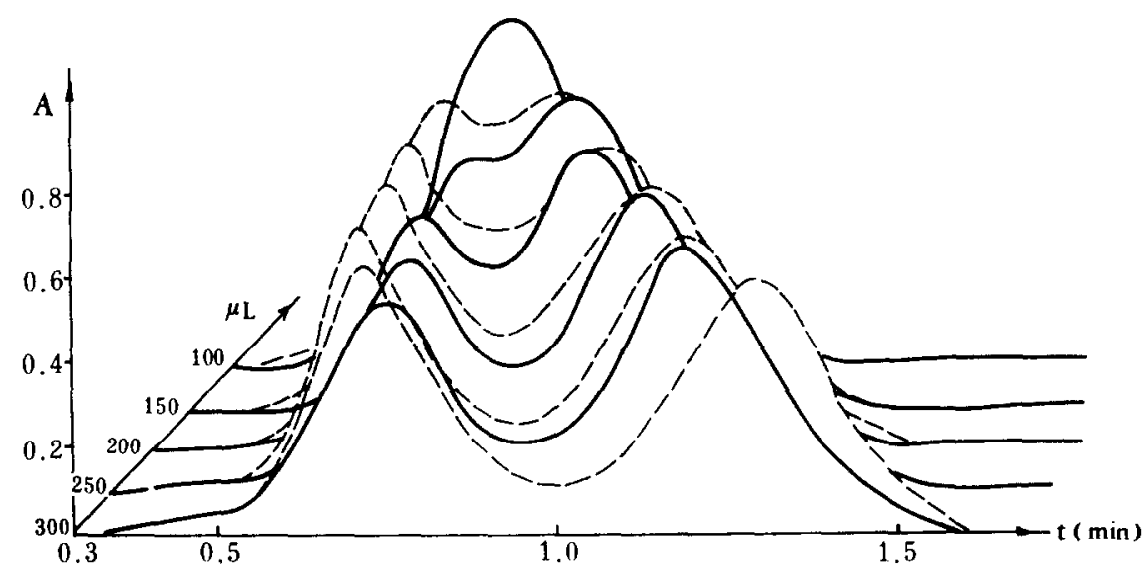

Fig. 6. Effect of injection volumes on the peak shape in SI (dashed line) and RI (solid line). 


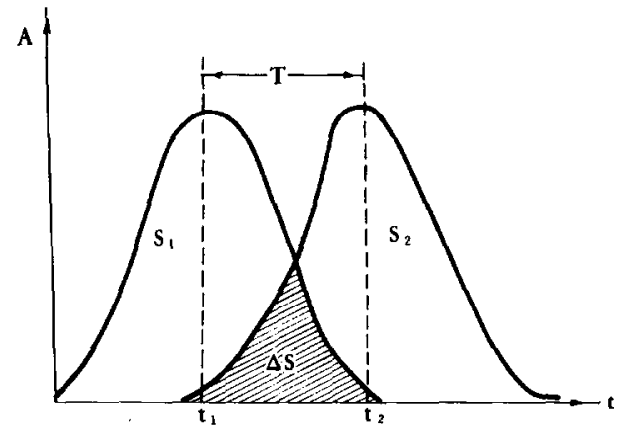

Fig. 8. Effect of overlap coefficient of double peaks on the sensitivity of determination. $t=$ Residence time; $T=$ the difference of two residence times.

In which $A$ is the absorbance, $A_{0}$ is the maximum absorbance of a peak and $\sigma$ is a constant. The overlap area and the overlap coefficient can be obtained when the curves are integrated, and they are expressed by Eq. 7 and Eq. 8, respectively

$$
\begin{aligned}
& \Delta S=2 A_{0} \exp [-T / 2 \sigma] \\
& S=\exp [-T / 2 \sigma] \times 100 \%
\end{aligned}
$$

If $S r$ and $S s$ represent the overlap coefficient of the front and the rear peaks in the RI and the SI respectively, then

$$
\begin{aligned}
S r / S s & =\exp [-(T r-T s) / 2 \sigma] \\
& =\exp \left[\left(\Delta \Phi_{1}+\Delta \Phi_{2}\right) / 2 \sigma\right]
\end{aligned}
$$

From Eq. 9 it can be found that the greater is the difference in residence time between the two chasing

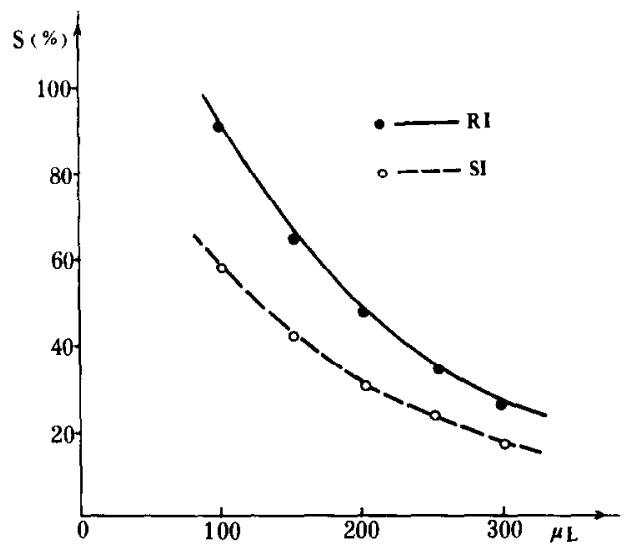

Fig. 9. Effect of injection volume on overlap coefficient in SI (dashed line) and RI (solid line).

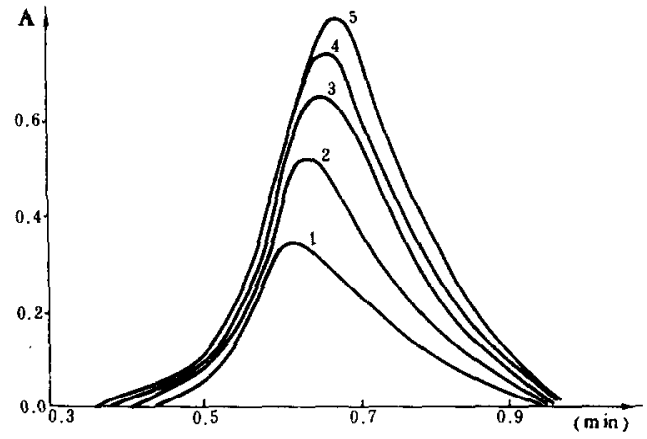

Fig. 10. Effect of the phen concentration on the peak shape in the $S \rightarrow R$ chasing model. Curves $1-5$ are $2,4,6,8$ and $10 \times 10^{-4} \mathrm{M}$ phen, respectively.

models of $R \rightarrow S$ and $S \rightarrow R$ in FLA, the higher is the sensitivity of the RI over that of SI. The overlap coefficient of the double peaks in SI and RI are shown in Fig. 9 as a function of the injection volume. When $\sigma=0.173, \Delta \Phi_{1}=0.04 \mathrm{~min}, \Delta \Phi_{2}=$ $0.12 \mathrm{~min}$ in the present study, $S r / S s=1.59$, so the overlap coefficient in RI is 1.59 times as large as that in SI.

\subsection{Effect of reagent concentration and flow rate on} residence time and difference in residence time

The effect of reagent concentration on peak shape in the $S \rightarrow R$ chasing model is shown in Fig. 10. Fig. 10 indicates that increasing the reagent concentration results in an increase of residence time, which can be seen in Fig. 11 more clearly. On the contrary, increasing the reagent concentration in the $R \rightarrow S$ chas-

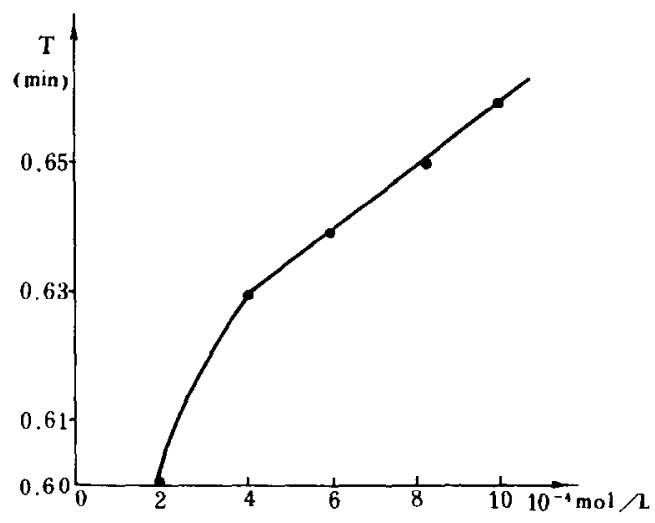

Fig. 11. Effect of the phen concentration on residence time. 


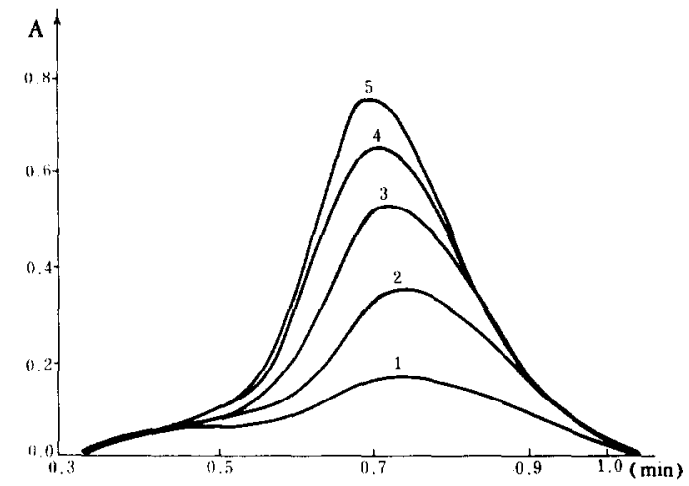

Fig, 12. Effect of the phen concentration on the peak shape in the $\mathrm{R} \rightarrow \mathrm{S}$ chasing model. Curves $1-5$ are 2, 4, 6, 8 and $10 \times 10^{-4} \mathrm{M}$ phen, respectively.

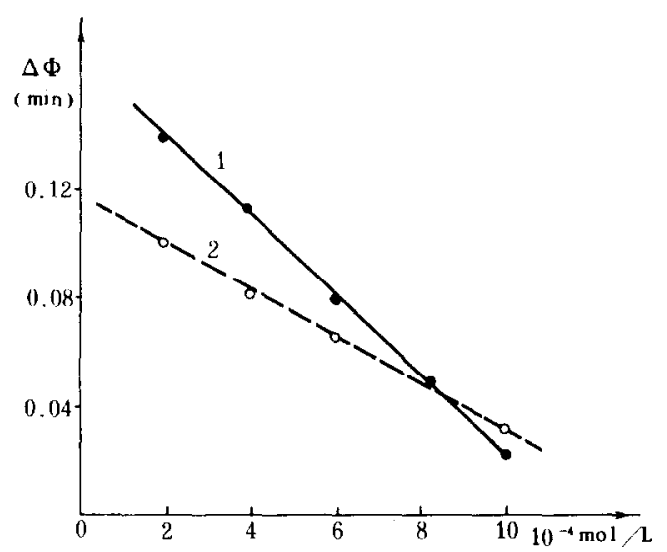

Fig. 13. Effect of the phen concentration on the difference in residence at different flow rates. The flow rates for curve 1 and curve 2 were 1.2 and $1.0 \mathrm{ml} / \mathrm{min}$, respectively.

ing mode results in a insignificant decrease of residence time as shown in Fig. 12. The effect of reagent concentration on the difference in residence time at different flow rates is shown in Fig. 13, which indicates that the difference in residence time, resulted from the two chasing modes, decreases with the increase of concentration but is always a positive value. This fact suggests that the diffusivities of reagent and sample were the parameter which affects the peak shape and the residence time. The concentration of a reagent or a sample can change the residence time but has less effect when compared to the effect of diffusivity.

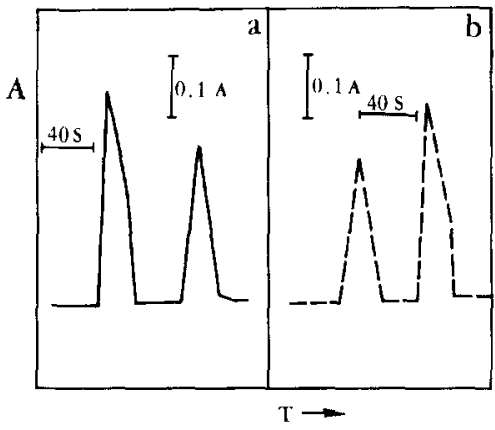

Fig. 14. Signals obtained in the $\mathrm{IO}^{3-}+\mathrm{I}^{-}+\mathrm{H}^{+}$reaction system. (a) $\mathrm{I}^{-}$was injected into the $\mathrm{IO}^{3-}+\mathrm{H}^{+}$solution. (b) $\mathrm{IO}^{3-}+\mathrm{H}^{+}$ was injected into the $\mathrm{I}^{-}$solution.

3.5. Explaining experimental results from the literature by using the difference in residence time

Fogg et al. [3] have studied the shape of the signal peak in $\mathrm{SI}$ and $\mathrm{RI}$ using the $\mathrm{IO}^{3}+\mathrm{I}^{-}+\mathrm{H}^{+}$reaction system. In their study, 24 sets of results were reported. All the peak shapes based on there results can be explained by the principle of difference in residence time proposed in this present paper. For example, the results obtained where $\mathrm{I}^{--}$was injected into $\mathrm{IO}^{3-}+\mathrm{H}^{+}$, and $\mathrm{IO}^{3-}+\mathrm{H}^{+}$was injected into $\mathrm{I}^{-}$ are shown in Fig. 14. Because $\mathrm{IO}^{3-}+\mathrm{H}^{+}$has a smaller diffusion coefficient than $\mathrm{I}^{-}$, and because a difference in residence time in the front and the rear peaks exists, the distance between the double peaks obtained when $\mathrm{IO}^{3-}+\mathrm{H}^{+}$was injected into $\mathrm{I}^{-}$is less than that obtained when $\mathrm{I}^{-}$was injected into $\mathrm{IO}^{3-}+\mathrm{H}^{+}$.

\section{Conclusions}

In summary it may be stated that:

(1) In a single channel FIA system, the peak shape and the phase difference are affected by the relation position of reagent and sample in the direction of the flow.

(2) Due to the different diffusivity of the reagent and sample, the peaks resulted from the two chasing models of $S \rightarrow R$ and $R \rightarrow S$ have a different residence time. The difference depends on the dispersion coefficients and concentrations of the reactants.

(3) If peak height is measured in FIA, the sensi- 
tivity increases with decreasing difference in residence time of the absorbance peaks formed at the two reagents/sample interface.

(4) When the concentration ratio of reagent and sample is approximately equals to the molar ratio of the complex compound, the sensitivity in RI is higher than that in SI if peak height was measured, while the sensitivity in RI is the same as that in SI if peak area was measured.

(5) The mode of injecting a reactant with less diffusivity into a reactant with larger diffusivity has a higher sensitivity than the mode of injecting a reaction with larger diffusivity into a reactant with less diffusivity.

\section{References}

[1] J. Ruzicka and E.H. Hansen, Anal. Chim. Acta, 78 (1975) 78. [2] K.S. Johnson and R.L. Pettey, Anal. Chem., 54 (1982) 1185.

[3] A.G. Fogg, X. Wang and J.F. Tyson, Analyst, 114 (1989) 1119.

[4] Shanghai Chemical Engineering College, Tianjin University and Zhejiong University, Chemical Engineering, 2nd edn. Chemical Industry, Beijing, 1980, p. 81.

[5] O. Levenspiel, Chemical Reaction Engineering, 2nd edn., Wiley, New York, 1972, Chap. 9.

[6] G. Chen, Chemical Reaction Engineering, 2nd edn., Chemical Industry, Beijing, 1990. 